\title{
OPEN Fibrinogen-Albumin-Ratio is an independent predictor of thromboembolic complications in patients undergoing VA-ECMO
}

Sebastian Roth ${ }^{1}$, Catrin Jansen ${ }^{1}$, René M'Pembele $^{1}$, Alexandra Stroda ${ }^{1}$, Udo Boeken ${ }^{2}$, Payam Akhyari ${ }^{2}$, Artur Lichtenberg ${ }^{2 \varpi}$, Markus W. Hollmann ${ }^{3}$, Ragnar Huhn ${ }^{1}$, Giovanna Lurati Buse ${ }^{1,4}$ \& Hug Aubin ${ }^{2,4}$

Veno-arterial extracorporeal membrane oxygenation (VA-ECMO) supports patients suffering from refractory cardiogenic shock. Thromboembolic complications (TeC) are common in VA-ECMO patients and are associated with increased morbidity and mortality. Valid markers to predict TeC in VA-ECMO patients are lacking. The present study investigated the predictive value of baseline FibrinogenAlbumin-Ratio (FAR) for in-hospital TeC in patients undergoing VA-ECMO. This retrospective cohort study included patients who underwent VA-ECMO therapy due to cardiogenic shock at the University Hospital Duesseldorf, Germany between 2011 and 2018. Main exposure was baseline FAR measured at initiation of VA-ECMO therapy. The primary endpoint was the in-hospital incidence of TeC. In total, 344 patients were included into analysis $(74.7 \%$ male, mean age $59 \pm 14$ years). The in-hospital incidence of TeC was $34 \%$. Receiver operating characteristics (ROC) curve of FAR for in-hospital TeC revealed an area under the curve of 0.67 [95\% confidence interval (CI) 0.61-0.74]. Youden index determined a cutoff of 130 for baseline FAR. Multivariate logistic regression revealed an adjusted odds-ratio of 3.72 [95\% $\mathrm{Cl}$ 2.26-6.14] for the association between FAR and TeC. Baseline FAR is independently associated with in-hospital TeC in patients undergoing VA-ECMO. Thus, FAR might contribute to the prediction of $\mathrm{TeC}$ in this cohort.

Venoarterial extracorporeal membrane oxygenation (VA-ECMO) is used to temporarily support the cardiac cycle and gas exchange in patients with acute cardiorespiratory failure ${ }^{1-3}$. Despite of continuous improvements in oxygenators and pump technologies, VA-ECMO therapy is still associated with a high rate of complications ${ }^{4-6}$. Previous studies state a mortality rate between 40 and $60 \%$. The incidence of thromboembolic complications $(\mathrm{TeC})$ such as ischemic stroke, cannula-associated deep vein thrombosis or arterial thromboembolism is estimated at $33 \%, 41 \%$ and $14 \%$ respectively ${ }^{9}$. These data show clearly that further improvement of VA-ECMO therapy is warranted. One approach is to identify prognostic biomarkers, for example to predict thromboembolic events in advance ${ }^{10}$. This could possibly improve the outcome due to early identification of therapeutic measures and potential treatment targets. In addition, the use of valid biomarkers could help to understand which patients could really benefit from VA-ECMO.

The Fibrinogen-Albumin-Ratio (FAR) has been suggested as an indicator for disease severity during prothrombotic conditions ${ }^{11-13}$. Fibrinogen and Albumin both have effects on blood clotting. While Fibrinogen is a clotting factor that elevates the aggregation of thrombocytes ${ }^{14}$, Albumin plays a role in inhibiting the function of thrombocytes and thrombus formation ${ }^{15,16}$. So far, data on the prognostic value of FAR for patients with VAECMO are scarce. A retrospective cohort study revealed that an elevated FAR within the first $24 \mathrm{~h}$ after initializing VA-ECMO therapy was associated with a higher risk of ischemic stroke ${ }^{17}$. To our best knowledge, the association

\footnotetext{
${ }^{1}$ Department of Anesthesiology, Medical Faculty and University Hospital Duesseldorf, Heinrich-Heine-University Duesseldorf, Moorenstr. 5, 40225 Duesseldorf, Germany. ${ }^{2}$ Department of Cardiac Surgery, Medical Faculty and University Hospital Duesseldorf, Heinrich-Heine-University Duesseldorf, Moorenstr. 5, 40225 Duesseldorf, Germany. ${ }^{3}$ Department of Anesthesiology, Amsterdam University Medical Center (AUMC), Location AMC, Meibergdreef 9, 1105 AZ Amsterdam, The Netherlands. ${ }^{4}$ These authors contributed equally: Giovanna Lurati Buse and Hug Aubin. ${ }^{\varpi}$ email: Artur.Lichtenberg@med.uni-duesseldorf.de
} 
between FAR and $\mathrm{TeC}$ in general has not been investigated yet. Therefore, we aimed to determine whether early FAR is associated with in-hospital TeC in patients undergoing VA-ECMO therapy.

\section{Methods}

This retrospective, single-center cohort study was conducted according to the guidelines for good clinical practice (GCP) and the declaration of Helsinki. The study was approved by the ethical committee of the Heinrich-HeineUniversity, Duesseldorf, Germany (reference number 5141R). All patients gave written informed consent to be registered in a dedicated database. This manuscript follows the STROBE reporting guidelines for retrospective cohort studies.

Participants. The present study included all patients who underwent extracorporeal life support (ECLS) at the University Hospital Duesseldorf, Germany between 2011 and 2018 due to refractory cardiogenic shock. Exclusion criteria were missing data regarding the primary endpoint, incomplete medical records so that documentation of $\mathrm{TeC}$ was not possible, age $<18$ years and the use of veno-venous ECMO. Anticoagulation was performed with unfractionated heparin according to the local standard or with argatroban, if appropriate. Anticoagulation monitoring was based on activated partial thromboplastin time (aPTT) or anti-factor Xa-activity.

Definition and assessment of main exposure. Main exposure was FAR on the day of initiation of VA-ECMO therapy. Measurements of Fibrinogen and Albumin values were performed in the central laboratory of the University Hospital Duesseldorf. The FAR was calculated by dividing Fibrinogen to Albumin ${ }^{12,17}$. Furthermore, baseline Fibrinogen and baseline Albumin were investigated alone to see if the ratio of both values is superior to the single values. In an additional analysis, FAR on day five was analysed as alternative exposure. The decision to choose these two time points for analysis was based on the rationale that VA-ECMO initiation itself might be associated with $\mathrm{TeC}$, for example due to cannula associated thrombosis. Hence, the predictive value of FAR before or after start of VA-ECMO therapy might be different. Day five was chosen based on local experiences suggesting that this might be a typical point of time where clinicians often have to decide about prognosis and whether therapy should be continued or not.

Outcome assessment. The primary endpoint of this study was the incidence of in-hospital TeC. TeC were defined as a composite of non-fatal arterial thrombosis or embolism, non-fatal venous thrombosis or embolism, non-fatal ischemic stroke, non-fatal myocardial infarction, or thromboembolic vascular mortality ${ }^{18}$. Arterial and venous thromboembolisms were defined as any new and symptomatic non-cardiac and non-cerebral arterial or venous thrombosis or embolism not causing death. Non-fatal myocardial infarction was defined according to the fourth universal definition of myocardial infarction ${ }^{19}$. Non-fatal ischemic stroke was defined according to the guidelines by the American Stroke Association ${ }^{20}$. Thromboembolic vascular mortality was defined as any $\mathrm{TeC}$ causing death. Data on $\mathrm{TeC}$ were extracted from electronic medical charts by personnel trained in the study definitions. TeC was confirmed when there was a clearly documented diagnosis that was approved by a physician specialized in intensive care medicine. Plausibility checks were done whenever further source documents were available.

Sample size. Due to the nature of this retrospective exploratory data analysis, we did not conduct formal sample size calculation. However, based on the current literature, we expected $\mathrm{TeC}$ in approximately $30 \%$ of patients ${ }^{8}$. With an estimated study sample of 350 patients, we expected approximately 105 thromboembolic events. This allowed to include up to 10 predefined co-variables for multivariable adjustment (see "Statistical analysis"). As 117 events could be observed in this study, we were able to add two further covariates (= 12 covariates in total) to a separate analysis that was conducted post factum during review process.

Statistical analysis. Patient characteristics are presented as absolute values with corresponding percentages for categorical data or as mean \pm standard deviation for continuous data, as appropriate. Shapiro-Wilks test was used to test for normal distribution of data. Fisher exact test and t-tests were used to test for differences between categorical and dichotomous data. Discrimination of baseline FAR for in-hospital $\mathrm{TeC}$ was analyzed by receiver operating characteristics (ROC) curve and the "area under the curve" (AUC). ROC analysis was also done for Fibrinogen and Albumin alone. De Long-Test was performed to compare ROC curves. A cutoff value for FAR was determined by Youden Index. Multivariable logistic regression analysis was used to assess the independent association (Odds ratio (OR); 95\% confidence interval (CI)) between elevated FAR and in-hospital TeC after adjustment by the following predefined covariables (forced entry): age, sex, chronic coronary syndrome, history of ischemic stroke, history of pulmonary embolism, arterial hypertension, diabetes mellitus, days of VA-ECMO therapy, continuous veno-venous hemodialysis treatment during hospitalization. The choice of covariates was based on literature research $\mathrm{h}^{2,3,21-23}$ and/or clinical experiences so that covariates were included if an association with $\mathrm{TeC}$ seemed possible. Baseline quick and baseline activated partial thromboplastin time (aPTT) could be added to an additional post factum logistic regression model. Model calibration was assessed using HosmerLemeshow-Test. Net reclassification index (NRI) and integrated discrimination index (IDI) were calculated for FAR and for Fibrinogen and Albumin alone. For all statistical tests, $p<0.05$ was considered significant. Analyses were performed with IBM SPSS Statistics version 26 (IBM, Armonk, New York, United States) and GraphPadPrism $^{\odot}$ statistical software version 6 (GraphPad software Inc, San Diego, California, United States). 


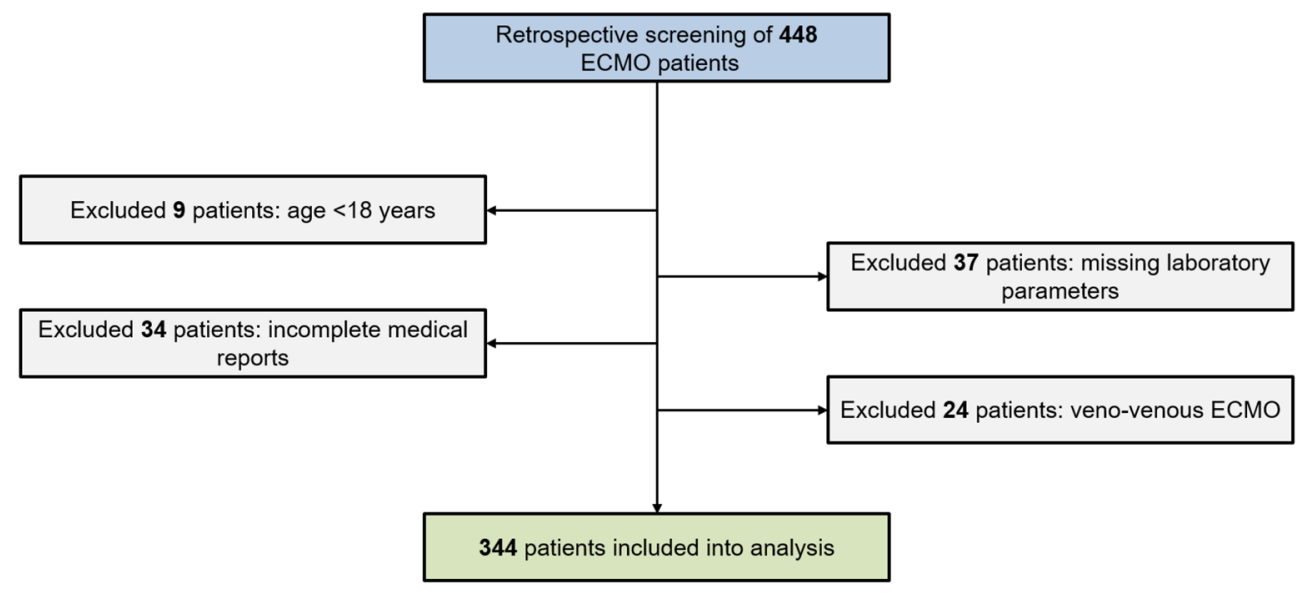

Figure 1. Study flow chart.

Ethics approval and consent to participate. The study was approved by the ethical committee of the Heinrich-Heine-University, Duesseldorf, Germany (reference number 5141R). All patients gave written informed consent to be registered in a dedicated database.

\section{Results}

Study cohort and baseline characteristics. The study flow chart is shown in Fig. 1. Of the included 344 patients, $257(74.7 \%)$ were male, the mean age was $59 \pm 14$. Table 1 reports detailed patients characteristics of the whole cohort and by primary outcome. In total, 117 patients $(34 \%)$ had a TeC during their hospital stay. The most common TeCs were arterial thromboembolic events $(63$ patients $=18.3 \%)$ and ischemic stroke $(40$ patients $=11.6 \%)$. Overall in-hospital mortality was 58.1\% (200/344). Patients with TeC during hospitalization had a significantly higher FAR (158 \pm 96$)$ than patients without TeC $(108 \pm 62)$ (see Fig. 2). Table 2 summarizes characteristics by FAR above and below the cut-off established by Youden Index. Patients with FAR $<130$ had significantly more major bleedings $(122(52.8 \%)$ versus $44(38.9 \%))$ and a higher rate of acute kidney injury requiring renal replacement therapy $(132(57.1 \%)$ versus $78(69.0 \%))$. In addition, patients with FAR below the calculated cutoff had significantly fewer non-fatal myocardial infarctions (3 (1.3\%) versus $8(7.1 \%))$ and other non-fatal arterial thromboembolisms $(29(12.6 \%)$ versus $34(30.1 \%))$. Furthermore, patients with FAR $<130$ had a significantly lower baseline quick value $(45 \pm 20 \%$ versus $52 \pm 20 \%)$.

ROC analysis and determination of cutoff. ROC analysis for baseline FAR and in-hospital TeC revealed an AUC of 0.67 [95\% CI 0.61-0.74; p<0.0001] (see Fig. 3). On day five, data for 212 patients were still available. ROC analysis for FAR on day five and in-hospital TeC revealed an AUC of 0.66 [95\% CI 0.57-0.76; $\mathrm{p}<0.0001$. Youden index determined a cutoff of 130 for baseline FAR. ROC analysis for baseline Fibrinogen alone and baseline Albumin alone revealed an AUC of 0.61 [95\% CI 0.54-0.67; p=0.001] and 0.61 [95\% CI 0.54-0.67; $\mathrm{p}=0.001$ ], respectively (see Supplementary Fig. 1). Comparison of ROC curves revealed that AUC-FAR was significantly higher than AUC-Fibrinogen (difference between areas $=0.064$ [95\% CI 0.018-0.111), $\mathrm{p}=0.0066$ ] Difference between area of AUC-FAR and AUC-Albumin was 0.064 [95\% CI - 0.002 to 0.13), $\mathrm{p}=0.056$ ].

Multivariate binary logistic regression. Binary logistic regression analysis with multivariable adjustment for ten predefined co-variables revealed a significant association between baseline FAR and in-hospital $\mathrm{TeC}$ with an OR of 3.72 (95\% CI 2.26-6.14) (see Table 3). The OR for the association between FAR on day 5 and in-hospital TeC was 5.79 [95\% CI 2.41-13.89]. A post factum logistic regression model including aPTT and quick as further covariables (= 12 covariables in total) revealed no new significant or relevant findings (see Supplementary Table 1).

Net reclassification index and integrated discrimination index. The overall NRI of FAR was $40.9 \%$. FAR-NRI for events was 26.4\% [95\% CI 20.8-32.7\%; $\mathrm{p}<0.0001$ ] and FAR-NRI for non-events was $14.5 \%$ [95\% CI 8.7-22.2\%; $\mathrm{p}<0.0001]$. Calculation for Fibrinogen alone revealed an overall NRI of $30.1 \%$ with an Fibrinogen-NRI of 9.4\% [95\% CI 4.8-16.2\%; $\mathrm{p}=0.0013$ ] for events and 20.7\% [95\% CI 15.6-26.6\%; $\mathrm{p}<0.0001$ ] for non-events. Calculation for Albumin alone revealed an overall NRI of $22.1 \%$ with an Albumin-NRI of $11.1 \%$ [95\% CI 6.1-18.3\%; $\mathrm{p}=0.0004$ ] for events and 11\% [95\% CI 7.3-15.8\%; $\mathrm{p}<0.0001$ ] for non-events. The IDI for FAR was 0.074 [95\% CI 0.041-0.107; p $<0.0001]$ and IDI for Fibrinogen and Albumin alone was 0.053 [95\% CI $0.025-0.081 ; \mathrm{p}=0.0002$ ] and 0.033 [95\% CI 0.012-0.056; $\mathrm{p}=0.003$ ], respectively. 


\begin{tabular}{|c|c|c|c|c|}
\hline & $\begin{array}{l}\text { All VA-ECMO patients } \\
(\mathrm{N}=344)\end{array}$ & $\begin{array}{l}\text { Patients without TEC } \\
(\mathrm{N}=227)\end{array}$ & Patients with TEC $(\mathrm{N}=117)$ & p-value ${ }^{a}$ \\
\hline \multicolumn{5}{|l|}{ Baseline characteristics } \\
\hline Male sex no. (\%) & $257(74.7)$ & $167(73.6)$ & $90(76.9)$ & 0.516 \\
\hline Age (years) & $59 \pm 14$ & $58.7 \pm 14.9$ & $58.8 \pm 13.7$ & 0.930 \\
\hline Duration of ECMO (days) & $7.6 \pm 5.9$ & $6.7 \pm 5.2$ & $9.3 \pm 6.9$ & $<0.0001$ \\
\hline \multicolumn{5}{|l|}{ Comorbidities no. (\%) } \\
\hline Arterial hypertension & $111(32.3)$ & $73(32.2)$ & $38(32.5)$ & 0.999 \\
\hline Diabetes & $72(20.9)$ & $46(20.3)$ & $26(22.2)$ & 0.677 \\
\hline Chronic coronary syndrome & $176(51.2)$ & $123(54.2)$ & $53(45.3)$ & 0.139 \\
\hline Peripheral artery disease & $37(10.8)$ & $20(8.8)$ & $17(14.5)$ & 0.141 \\
\hline Prior MI & $162(47.1)$ & 99 (43.6) & $63(53.8)$ & 0.087 \\
\hline Prior stroke & $22(6.4)$ & $14(6.2)$ & $8(6.8)$ & 0.819 \\
\hline Prior pulmonary embolism & $14(4.1)$ & $7(3.1)$ & $7(6.0)$ & 0.250 \\
\hline \multicolumn{5}{|l|}{ Clinical endpoints no. (\%) } \\
\hline In hospital death & $200(58.1)$ & $133(58.6)$ & $67(57.3)$ & 0.819 \\
\hline Major bleeding & $166(48.3)$ & $115(50.7)$ & $51(43.6)$ & 0.255 \\
\hline AKI with CVVHD & $210(61.0)$ & $127(55.9)$ & $83(70.9)$ & 0.007 \\
\hline $\begin{array}{l}\text { Thromboembolic complica- } \\
\text { tions no. (\%) }\end{array}$ & $117(34.0)$ & & & \\
\hline MI & $11(3.2)$ & $0(0)$ & $11(9.4)$ & $<0.0001$ \\
\hline Stroke & 40 (11.6) & $0(0)$ & $40(34.2)$ & $<0.0001$ \\
\hline Art. thromboembolism & $63(18.3)$ & $0(0)$ & $63(53.8)$ & $<0.0001$ \\
\hline Ven. thromboembolism & $11(3.2)$ & $0(0)$ & $11(9.4)$ & $<0.0001$ \\
\hline Vascular death & $3(0.9)$ & $0(0)$ & $3(2.6)$ & 0.039 \\
\hline \multicolumn{5}{|c|}{ Laboratory parameters at baseline } \\
\hline Creatinine $(\mathrm{mg} / \mathrm{dl})$ & $1.9 \pm 1.6$ & $1.9 \pm 1.5$ & $1.9 \pm 1.6$ & 0.944 \\
\hline Leukocytes $(\times 1000 / \mu \mathrm{l})$ & $14.5 \pm 7.5$ & $14.7 \pm 7.6$ & $14.1 \pm 7.3$ & 0.492 \\
\hline Hemoglobin $(\mathrm{g} / \mathrm{dl})$ & $10.7 \pm 2.3$ & $10.6 \pm 2.3$ & $10.8 \pm 2.4$ & 0.377 \\
\hline Hematocrite (\%) & $33 \pm 17$ & $33.3 \pm 20.6$ & $32.5 \pm 7.5$ & 0.688 \\
\hline Thrombocytes $(\times 1000 / \mu \mathrm{l})$ & $174 \pm 97$ & $171 \pm 90$ & $179 \pm 110$ & 0.477 \\
\hline aPTT (s) & $76 \pm 47$ & $77 \pm 48$ & $73 \pm 46$ & 0.510 \\
\hline Quick (\%) & $48 \pm 21$ & $47 \pm 21$ & $50 \pm 20$ & 0.151 \\
\hline Antithrombin III (\%) & $55 \pm 32$ & $58 \pm 36$ & $52 \pm 20$ & 0.184 \\
\hline D-Dimer & $16.2 \pm 21.8$ & $15 \pm 20$ & $19 \pm 25$ & 0.221 \\
\hline Fibrinogen $(\mathrm{mg} / \mathrm{dl})$ & $288 \pm 149$ & $269 \pm 139$ & $326 \pm 162$ & 0.001 \\
\hline Albumin $(\mathrm{g} / \mathrm{l})$ & $2.5 \pm 0.8$ & $2.6 \pm 0.8$ & $2.3 \pm 0.8$ & 0.001 \\
\hline FAR & $125 \pm 79$ & $108 \pm 62$ & $158 \pm 96$ & $<0.0001$ \\
\hline
\end{tabular}

Table 1. Baseline characteristics of the whole cohort and in patients without and with TeC. Data are presented as mean \pm standard deviation or as absolute values with percentages, as appropriate. TEC thromboembolic complication, $A K I$ acute kidney injury, aPTT activated partial thromboplastin time, CVVHD continuous Veno-venous hemodialysis, VA-ECMO veno-arterial extracorporeal membrane oxygenation, FAR FibrinogenAlbumin ratio, $M I$ myocardial infarction. a $p$ value of Chi-square test or two-tailed unpaired t-test after Levene's test for equality of variances.

\section{Discussion}

The main finding of the present study is that baseline FAR is independently associated with in-hospital TeC in patients requiring VA-ECMO due to refractory cardiogenic shock. Furthermore, this study identified a cutoff of 130 for baseline FAR, which was related to a higher likelihood of TeC. The independent association between FAR and TeC was also present when FAR was measured on day 5 of VA-ECMO therapy.

Prediction and prevention of TeC in VA-ECMO patients. One of the most important issues in terms of treating VA-ECMO patients is to understand which patients could really benefit from VA-ECMO. This decision has to be faced prior to the initiation of VA-ECMO. Once initiated, another important question in terms of prognosis and risk stratification is to decide whether VA-ECMO therapy should be continued or limited, for example if patients suffer from severe complications.

In the last decade, several scores such as the Survival after Veno-Arterial ECMO (SAVE) score ${ }^{21}$ have been suggested to help clinicians with these issues, but data focused on the prediction of $\mathrm{TeC}$ are rare. In a small retrospective cohort study with 62 patients, Trudzinski and colleagues tried to find predictors for TeC in patients 


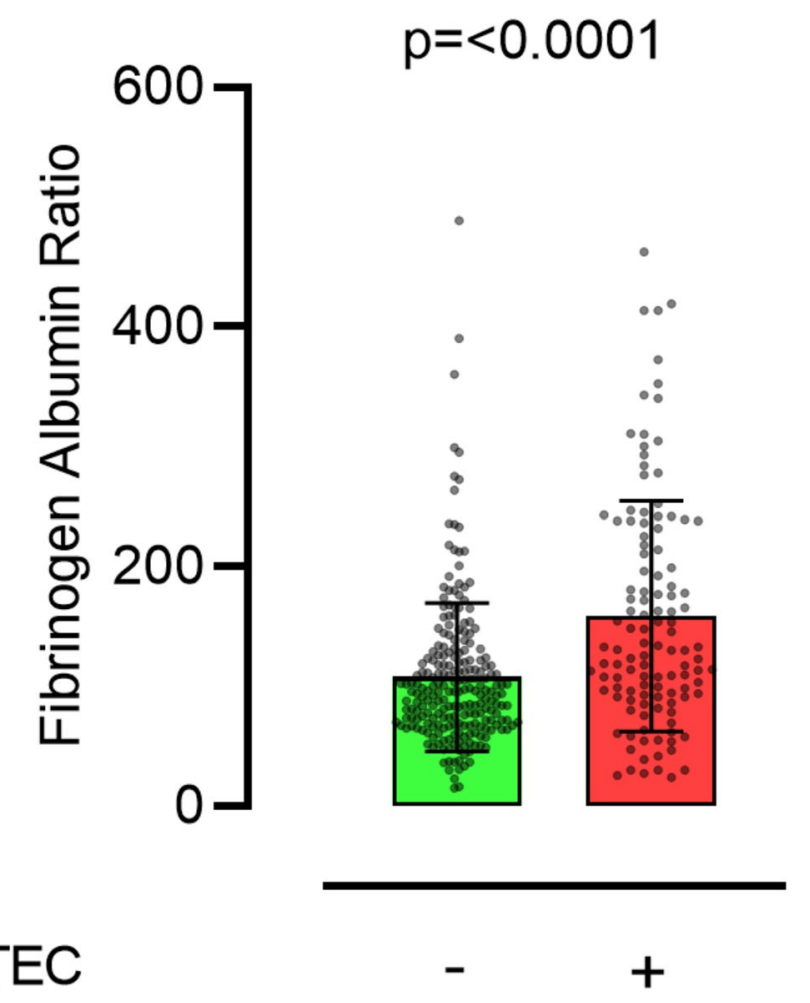

Figure 2. Box plot shows that Fibrinogen-Albumin-Ratio levels in patients with or without thromboembolic complication (TEC) are significantly different.

undergoing veno-venous ECMO due to respiratory failure. This study found that the quality of anticoagulation and ECMO runtime predicted thromboembolic events ${ }^{22}$. Most other studies in this field also focused on the role of anticoagulation and the monitoring of coagulation parameters such as activated clotting time (ACT), aPTT or anti-factor Xa-activity ${ }^{24,25}$. Pieri et al. performed a small case-control study with a total of 20 patients to compare bivalirudin-based anticoagulation with heparin-based protocols in a population of patients treated with VV-ECMO or VA-ECMO with a target aPTT of 45-60 $\mathrm{s}^{26}$. The authors concluded that Bivalirudin-based anticoagulation may represent a new method of anticoagulation for reducing thromboembolic complications. A recently published study by Fisser and colleagues investigated Argatroban versus heparin in patients without heparin-induced thrombocytopenia during VV-ECMO ${ }^{27}$. This prospective cohort study included 465 patients and found out that Argatroban was non-inferior to Heaprin regarding bleeding and thrombosis. In summary, data regarding anticoagulation and monitoring of coagulation parameters are still inconclusive.

FAR to predict TeC in VA-ECMO patients. Regarding pathophysiologic mechanisms behind the association of FAR and $\mathrm{TeC}$, Albumin is an essential plasma protein that has been proposed to be related to inflammatory and hemostatic processes ${ }^{11}$. Moreover, Albumin plays a role in the inhibition of platelet activation ${ }^{15}$. Fibrinogen on the other hand is an indicator of a procoagulatory status and contributes to inflammation at diverse levels ${ }^{12,14}$. The combination of these characteristics served as a basis to hypothesize that the ratio of Fibrinogen and Albumin may predict TeC in VA-ECMO patients as this cohort is at high risk for TeC. To date-to our best knowledge-there is only one study by Acharya and colleagues that investigated the predictive value of FAR in patients undergoing VA-ECMO ${ }^{17}$. In a retrospective single-center cohort study, this study analysed 157 patients regarding FAR measured within the first $24 \mathrm{~h}$ of VA-ECMO therapy and determined its prognostic value for the incidence of in-hospital ischemic stroke ${ }^{17}$. This study showed a significant association between an elevated FAR (>125) and in-hospital ischemic stroke. Our results add to these data by not only investigating the association between FAR and ischemic stroke, but with $\mathrm{TeC}$ in general. In addition, our data reveal a very similar cutoff for FAR (=130) so that this cutoff seems to be suitable. Finally, our study had a larger sample size (344 vs. 157).

Referring to patient characteristics of our study, overall in-hospital mortality rate was $58.1 \%$. This is in line with previously published data ${ }^{2}$. Interestingly, mortality was not influenced by $\mathrm{TeC}$ (see Table 1 ). However, it is important to mention that similar mortality rates do not automatically mean that there was no life impact. Unfortunately, we cannot provide data on more patient-centered outcomes such as "days alive and out of hospital" 28 . Another remarkable finding was that duration of VA-ECMO was significantly different between patients with or without $\mathrm{TeC}(9.3 \pm 6.9$ vs. $6.7 \pm 5.2)$. This aspect is underlined by the results of multivariate analysis, which also showed that the length of VA-ECMO therapy was independently associated with TeC (OR 1.08 [95\% CI 1.04-1.13]. Thus, taken together, days of VA-ECMO seem to be a relevant risk factor to develop TeC. 
Baseline characteristics

\begin{tabular}{|c|c|c|c|}
\hline Male sex no. $(\%)$ & $169(73.2)$ & $88(77.9)$ & 0.359 \\
\hline Age (years) & $58 \pm 15$ & $59 \pm 14$ & 0.788 \\
\hline Duration of ECMO (days) & $7.5 \pm 5.9$ & $7.7 \pm 6.0$ & 0.777 \\
\hline \multicolumn{4}{|l|}{ Comorbidities no. $(\%)$} \\
\hline Arterial hypertension & $76(32.9)$ & $35(31.0)$ & 0.806 \\
\hline Diabetes & $49(21.2)$ & $23(20.4)$ & 0.889 \\
\hline Chronic coronary syndrome & $121(52.4)$ & $55(48.7)$ & 0.566 \\
\hline Peripheral artery disease & $26(11.3)$ & $11(9.7)$ & 0.715 \\
\hline Prior MI & $109(47.2)$ & $53(46.9)$ & 0.999 \\
\hline Prior stroke & $16(6.9)$ & $6(5.3)$ & 0.646 \\
\hline Prior pulmonary embolism & $9(3.9)$ & $5(4.4)$ & 0.779 \\
\hline \multicolumn{4}{|l|}{ Clinical endpoints no. (\%) } \\
\hline In hospital death & $135(58.4)$ & $65(57.5)$ & 0.908 \\
\hline Major bleeding & $122(52.8)$ & $44(38.9)$ & 0.016 \\
\hline AKI with CVVHD & $132(57.1)$ & $78(69.0)$ & 0.035 \\
\hline Thromboembolic complications no. (\%) & $56(24.2)$ & $61(54.0)$ & $<0.0001$ \\
\hline MI & $3(1.3)$ & $8(7.1)$ & 0.007 \\
\hline Stroke & $23(10.0)$ & $17(15.0)$ & 0.209 \\
\hline Art. thromboembolism & $29(12.6)$ & $34(30.1)$ & $<0.0001$ \\
\hline Ven. thromboembolism & $8(3.5)$ & $3(2.7)$ & 0.999 \\
\hline Vascular death & $1(0.4)$ & $2(1.8)$ & 0.252 \\
\hline \multicolumn{4}{|l|}{ Laboratory parameters at baseline } \\
\hline Creatinine $(\mathrm{mg} / \mathrm{dl})$ & $1.9 \pm 1.5$ & $1.6 \pm 1.5$ & 0.439 \\
\hline Leukocytes $(\times 1000 / \mu \mathrm{l})$ & $14.5 \pm 7.6$ & $14.5 \pm 7.3$ & 0.965 \\
\hline Hemoglobin $(\mathrm{g} / \mathrm{dl})$ & $10.8 \pm 2.4$ & $10.4 \pm 2.0$ & 0.122 \\
\hline Hematocrite (\%) & $33 \pm 8$ & $34 \pm 28$ & 0.424 \\
\hline Thrombocytes $(\times 1000 / \mu \mathrm{l})$ & $171 \pm 93$ & $180 \pm 106$ & 0.451 \\
\hline aPTT (s) & $79 \pm 48$ & $69 \pm 44$ & 0.087 \\
\hline Quick (\%) & $45 \pm 20$ & $52 \pm 20$ & 0.003 \\
\hline Antithrombin III (\%) & $56 \pm 36$ & $54 \pm 20$ & 0.624 \\
\hline D-Dimer & $17.2 \pm 22.0$ & $14.5 \pm 21.2$ & 0.299 \\
\hline Fibrinogen (mg/dl) & $222 \pm 87$ & $423 \pm 161$ & $<0.0001$ \\
\hline Albumin $(\mathrm{g} / \mathrm{l})$ & $2.7 \pm 0.8$ & $2.1 \pm 0.6$ & $<0.0001$ \\
\hline FAR & $82.5 \pm 26.7$ & $212.2 \pm 78.9$ & $<0.0001$ \\
\hline
\end{tabular}

Table 2. Baseline characteristics in patients with FAR $<130$ and $\geq 130$. Data are presented as mean \pm standard deviation or as absolute values with percentages, as appropriate. AKI acute kidney injury, aPTT activated partial thromboplastin time, $C V V H D$ continuous Veno-venous hemodialysis, ECMO extracorporeal membrane oxygenation, FAR Fibrinogen-Albumin ratio, $M I$ myocardial infarction. ${ }^{\text {a }} p$ value of Chi-square test or two-tailed unpaired t-test after Levene's test for equality of variances.

\section{Strengths and limitations}

This study has several strengths. One strength of this study is that there was a high number of events $(\mathrm{TeC}$ : $117 / 344=34 \%)$ so that we could adjust for ten covariables in our multivariate logistic regression model (12 covariables post factum). Another strength is that all included patients were registered in a dedicated database, which ensured higher quality of our data.

This study also has limitations: first, this is a retrospective, single-center cohort study. However, baseline characteristics and in-hospital mortality rate $(58.1 \%)$ were in line with previously published data ${ }^{2}$, suggesting that both VA-ECMO indication and outcome in our centre might be representative for larger practice. Importantly, before drawing final conclusions, the predictive value of FAR for TeC should be investigated in prospective multicenter studies. Second, no cox regression could be performed as the exact time point of TeCs was not always documented in patients' medical records. Third, our database did not include reasons for initiation of VA-ECMO therapy. Although we know that all patients had refractory cardiogenic shock, we do not have information regarding the reason that led to cardiogenic shock. 


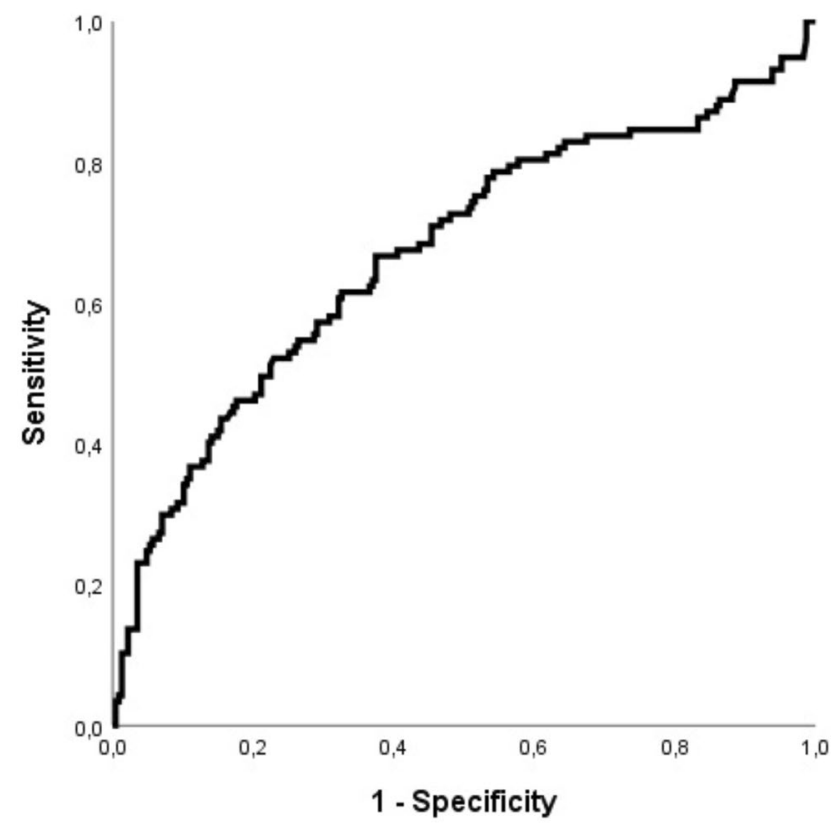

Figure 3. ROC curve shows moderate discrimination of baseline Fibrinogen-Albumin-Ratio for in-hospital thromboembolic complications (AUC $=0.67$ [95\% CI 0.61-0.74]).

\begin{tabular}{|l|l|l|l|c|}
\hline Covariables & Odds ratio & Lower 95\% CI & Upper 95\% CI & p-value \\
\hline FAR & 3.722 & 2.258 & 6.135 & $<0.001$ \\
\hline Sex & 0.814 & 0.464 & 1.428 & 0.472 \\
\hline Age & 1.004 & 0.986 & 1.022 & 0.667 \\
\hline CCS & 0.661 & 0.393 & 1.114 & 0.120 \\
\hline Prior Stroke & 1.066 & 0.392 & 2.895 & 0.900 \\
\hline Prior PE & 2.127 & 0.643 & 7.033 & 0.216 \\
\hline Diabetes mellitus & 1.255 & 0.669 & 2.354 & 0.479 \\
\hline Arterial hypertension & 0.886 & 0.504 & 1.558 & 0.674 \\
\hline CVVHD & 1.383 & 0.819 & 2.336 & 0.226 \\
\hline Days of VA-ECMO & 1.083 & 1.037 & 1.130 & $<0.001$ \\
\hline
\end{tabular}

Table 3. Multivariate logistic regression for the association between baseline Fibrinogen-Albumin-Ratio and thromboembolic complications. CCS Chronic coronary syndrome, CI Confidence Interval, CVVHD Continous Veno-Venous Hemodialysis, FAR Fibrinogen-Albumin-Ratio, PE Pulmonary Embolism, VA-ECMO VenoArterial Extracorporeal Membrane Oxygenation.

\section{Conclusions}

In conclusion, this study shows that baseline FAR is independently associated with in-hospital TeC in patients undergoing VA-ECMO. Therefore, FAR might be used to support the prediction of $\mathrm{TeC}$ in this cohort. Future studies should validate these findings with a prospective design.

\section{Data availability}

The datasets generated during and/or analysed during the current study are available from the first author on reasonable request.

Received: 10 June 2021; Accepted: 22 July 2021

Published online: 17 August 2021

\section{References}

1. Boeken, U. et al. Extracorporeal life support (ECLS)-Update 2020. Medizinische Klin. - Intensivmed. und Notfallmedizin 116, 56-58 (2021).

2. Kowalewski, M. et al. Venoarterial extracorporeal membrane oxygenation for postcardiotomy shock-Analysis of the extracorporeal life support organization registry. Crit. Care Med. (Publish Ah) (2021). 
3. Ponikowski, P. et al. 2016 ESC Guidelines for the diagnosis and treatment of acute and chronic heart failure. Eur. Heart J. 37, 2129-2200m (2016).

4. Aubin, H. et al. A suprainstitutional network for remote extracorporeal life support: A retrospective cohort study. JACC Hear. Fail. 4, 698-708 (2016).

5. Mehdiani, A. et al. Extracorporeal membrane oxygenation after heart transplantation: Impact of type of cannulation. Thorac. Cardiovasc. Surg. https://doi.org/10.1055/s-0039-3400472 (2020).

6. Bunte, S. et al. Bilirubin-A possible prognostic mortality marker for patients with ECLS. J. Clin. Med. 9, 1727 (2020).

7. Rao, P., Khalpey, Z., Smith, R., Burkhoff, D. \& Kociol, R. D. Venoarterial extracorporeal membrane oxygenation for cardiogenic shock and cardiac arrest. Circ. Heart Fail. 11, e004905 (2018).

8. Stretch, R., Sauer, C. M., Yuh, D. D. \& Bonde, P. National trends in the utilization of short-term mechanical circulatory support: Incidence, outcomes, and cost analysis. J. Am. Coll. Cardiol. 64, 1407-1415 (2014).

9. Fux, T., Holm, M., Corbascio, M., Lund, L. H. \& van der Linden, J. Venoarterial extracorporeal membrane oxygenation for postcardiotomy shock: Risk factors for mortality. J. Thorac. Cardiovasc. Surg. 156, 1894-1902.e3 (2018).

10. De Hert, S. G. \& Lurati Buse, G. A. Cardiac biomarkers for the prediction and detection of adverse cardiac events after noncardiac surgery: A narrative review. Anesth. Analg. 131, 187-195 (2020).

11. Zhang, D. P. et al. The Fibrinogen-to-Albumin Ratio is associated with outcomes in patients with coronary artery disease who underwent percutaneous coronary intervention. Clin. Appl. Thromb. 26, (2020).

12. An, Q., Liu, W., Yang, Y. \& Yang, B. Preoperative fibrinogen-to-albumin ratio, a potential prognostic factor for patients with stage IB-IIA cervical cancer. BMC Cancer 20, 1-8 (2020).

13. Bi, X. et al. Prediction of severe illness due to COVID-19 based on an analysis of initial Fibrinogen to Albumin Ratio and Platelet count. Platelets 31, 674-679 (2020).

14. Herrick, S., Blanc-Brude, O., Grey, A. \& Laurent, G. Fibrinogen. Int. J. Biochem. Cell Biol. 31, 741-746 (1999).

15. Mulvihill, J. N., Faradji, A., Oberling, F. \& Cazenave, J.-P. Surface passivation by human albumin of plasmaperesis circuits reduces platelet accumulation and thrombus formation. Experimental and clinical studies. J. Biomed. Mater. Res. 24, 155-163 (1990).

16. Thielmann, M. et al. Heart-type fatty acid binding protein and ischemia-modified albumin for detection of myocardial infarction after coronary artery bypass graft surgery. Ann. Thorac. Surg. 104, 130-137 (2017).

17. Acharya, P. et al. Fibrinogen albumin ratio and ischemic stroke during venoarterial extracorporeal membrane oxygenation. ASAIO J. https://doi.org/10.1097/MAT.0000000000000992 (2020).

18. Devereaux, P. J. et al. Dabigatran in patients with myocardial injury after non-cardiac surgery (MANAGE): An international, randomised, placebo-controlled trial. Lancet 391, 2325-2334 (2018).

19. Thygesen, K. et al. Fourth universal definition of myocardial infarction (2018). Eur. Heart J. 40, 237-269 (2019).

20. Kleindorfer, D. O. et al. 2021 Guideline for the Prevention of Stroke in Patients With Stroke and Transient Ischemic Attack: A Guideline From the American Heart Association/American Stroke Association. Stroke https://doi.org/10.1161/str.0000000000 000375 (2021).

21. Schmidt, M. et al. Predicting survival after ECMO for refractory cardiogenic shock: The survival after veno-arterial-ECMO (SAVE)-score. Eur. Heart J. 36, 2246-2256 (2015).

22. Trudzinski, F. C. et al. Runtime and aPTT predict venous thrombosis and thromboembolism in patients on extracorporeal membrane oxygenation: A retrospective analysis. Ann. Intensive Care 6, 2-9 (2016).

23. Loforte, A. et al. Role of intra-aortic balloon pump and extracorporeal membrane oxygenation in early graft failure after cardiac transplantation. Artif. Organs 40, E136-E145 (2016).

24. Parker, R. I. Anticoagulation monitoring during extracorporeal membrane oxygenation: Continuing progress. Crit. Care Med. 48, 1920-1921 (2020).

25. Delmas, C. et al. Anticoagulation monitoring under ECMO support: A comparative study between the activated coagulation time and the anti-Xa activity assay. J. Intensive Care Med. 35, 679-686 (2020).

26. Pieri, M. et al. Bivalirudin versus heparin as an anticoagulant during extracorporeal membrane oxygenation: A case-control study. J. Cardiothorac. Vasc. Anesth. 1, 30-34 (2013).

27. Fisser, C. et al. Argatroban versus heparin in patients without heparin-induced thrombocytopenia during venovenous extracorporeal membrane oxygenation: A propensity-score matched study. Crit. Care 1, 10. https://doi.org/10.1186/s13054-021-03581-x (2021).

28. Jerath, A., Austin, P. C. \& Wijeysundera, D. N. Days alive and out of hospital: Validation of a patient-centered outcome for perioperative medicine. Anesthesiology 131, 84-93 (2019).

\section{Author contributions}

S.R.: concept/design, data analysis/interpretation, statistics, writing of article. C.J.: data collection, data analysis/ interpretation, statistics, writing of article. R.M. and A.S.: data analysis/interpretation, statistics, critical revision of article. U.B., P.A., M.W.H. and R.H.: critical revision of article. A.L.: drafting article, critical revision of article. G.L.B and H.A.: concept/design, data analysis/interpretation, critical revision of article.

\section{Funding}

Open Access funding enabled and organized by Projekt DEAL.

\section{Competing interests}

The authors declare no competing interests.

\section{Additional information}

Supplementary Information The online version contains supplementary material available at https://doi.org/ 10.1038/s41598-021-95689-x.

Correspondence and requests for materials should be addressed to A.L.

Reprints and permissions information is available at www.nature.com/reprints.

Publisher's note Springer Nature remains neutral with regard to jurisdictional claims in published maps and institutional affiliations. 
(c) (i) Open Access This article is licensed under a Creative Commons Attribution 4.0 International cc) License, which permits use, sharing, adaptation, distribution and reproduction in any medium or format, as long as you give appropriate credit to the original author(s) and the source, provide a link to the Creative Commons licence, and indicate if changes were made. The images or other third party material in this article are included in the article's Creative Commons licence, unless indicated otherwise in a credit line to the material. If material is not included in the article's Creative Commons licence and your intended use is not permitted by statutory regulation or exceeds the permitted use, you will need to obtain permission directly from the copyright holder. To view a copy of this licence, visit http://creativecommons.org/licenses/by/4.0/.

(C) The Author(s) 2021 